OPEN ACCESS

Edited by:

Marion S. Röder,

Leibniz Institute of Plant Genetics and

Crop Plant Research (IPK),

Germany

Reviewed by:

Pasqualina Colasuonno,

University of Bari Aldo Moro,

Italy

Jian Ma,

Sichuan Agricultural University,

China

*Correspondence:

Matthew J. Hayden

matthew.hayden@agriculture.vic.gov.au

Urmil K. Bansal

urmil.bansal@sydney.edu.au

${ }^{\dagger}$ These authors have contributed equally to this work

Specialty section:

This article was submitted to

Plant Breeding,

a section of the journal

Frontiers in Plant Science

Received: 07 October 2019

Accepted: 20 December 2019

Published: 04 February 2020

Citation:

Nsabiyera V, Baranwal D, Qureshi N, Kay P, Forrest K, Valárik M, Doležel J,

Hayden MJ, Bariana HS and

Bansal UK (2020) Fine Mapping of

Lr49 Using 90K SNP Chip Array and

Flow-Sorted Chromosome

Sequencing in Wheat.

Front. Plant Sci. 10:1787.

doi: 10.3389/fpls.2019.01787

\section{Fine Mapping of Lr49 Using 90K SNP Chip Array and Flow-Sorted Chromosome Sequencing in Wheat}

\author{
Vallence Nsabiyera ${ }^{1 \dagger}$, Deepak Baranwal ${ }^{1 \dagger}$, Naeela Qureshi ${ }^{1,2}$, Pippa Kay ${ }^{2}$, Kerrie Forrest ${ }^{2}$, \\ Miroslav Valárik ${ }^{3}$, Jaroslav Doležel ${ }^{3}$, Matthew J. Hayden ${ }^{2,4 *}$, Harbans S. Bariana ${ }^{1}$ \\ and Urmil K. Bansal ${ }^{1 *}$ \\ 1 Faculty of Science, School of Life Sciences and Environment, The University of Sydney Plant Breeding Institute, Cobbitty, \\ NSW, Australia, ${ }^{2}$ Agriculture Victoria Research, AgriBio, Bundoora, VIC, Australia, ${ }^{3}$ Institute of Experimental Botany of the \\ Czech Academy of Sciences, Centre of the Region Haná for Biotechnological and Agricultural Research, Olomouc, Czechia, \\ ${ }^{4}$ School of Applied Systems Biology, La Trobe University, Bundoora, VIC, Australia
}

Leaf rust, caused by Puccinia triticina, threatens global wheat production due to the constant evolution of virulent pathotypes that defeat commercially deployed all stageresistance (ASR) genes in modern cultivars. Hence, the deployment of combinations of adult plant resistance (APR) and ASR genes in new wheat cultivars is desirable. Adult plant resistance gene Lr49 was previously mapped on the long arm of chromosome 4B of cultivar VL404 and flanked by microsatellite markers barc163 (8.1 cM) and wmc349 (10.1 $\mathrm{cM}$ ), neither of which was sufficiently closely linked for efficient marker assisted selection. This study used high-density SNP genotyping and flow sorted chromosome sequencing to fine-map the Lr49 locus as a starting point to develop a diagnostic marker for use in breeding and to clone this gene. Marker SunKASP_21 was mapped $0.4 \mathrm{cM}$ proximal to Lr49, whereas a group of markers including sunKASP_24 were placed $0.6 \mathrm{cM}$ distal to this gene. Testing of the linked markers on 75 Australian and 90 European cultivars with diverse genetic backgrounds showed that SunKASP_21 was most strongly associated with $L r 49$. Our results also show that the $L r 49$ genomic region contains structural variation relative to the reference stock Chinese Spring, possibly an inverted genomic duplication, which introduces a new set of challenges for the Lr49 cloning.

\footnotetext{
Keywords: adult plant resistance, chromosome sorting, Infinium iSelect 90K SNP array, leaf rust, marker assisted breeding
}

\section{HIGHLIGHTS}

High-density SNP genotyping and flow-sorted chromosome sequencing were used to fine map adult plant leaf rust resistance gene Lr49. 


\section{INTRODUCTION}

Leaf rust, caused by Puccinia triticina $(\mathrm{Pt})$, is one of the most important diseases of wheat worldwide and can result in yield losses of up to $70 \%$ (Kolmer, 2005). While wheat has inherent defense mechanisms to resist diseases, an emphasis on selection for high yield and other desirable traits has resulted in a narrow genetic base for disease resistance (Borlaug, 2007). The release of resistant cultivars is the best strategy to control leaf rust, and to reduce production costs and risk of environmental pollution resulting from fungicide usage (Bariana, 2003; Bariana et al., 2007; Bariana and Bansal, 2017).

Many leaf rust resistance genes have been identified and named in wheat (McIntosh et al., 1995; McIntosh et al., 2013; Bariana and Bansal, 2017). Resistance genes include two categories; all stage resistance (ASR) and adult plant resistance (APR). The ASR genes are effective throughout the life of the plant, whereas adult plant resistance genes are effective only at adult plant stage. A majority of the formally named genes confer ASR (McIntosh et al., 1995; McIntosh et al., 2013). ASR genes exhibit hypersensitive reaction to condition a high level of resistance against avirulent pathogen isolates. However, they are prone to breakdown when the pathogen evolves to acquire virulence. The ASR genes can be identified at the seedling stage under greenhouse conditions. In contrast, APR genes express at the postseedling stages and retard pathogen growth. They are considered durable due to their race nonspecific nature. Examples of pleiotropic APR genes include Lr34/Yr18 (Singh, 1992), Lr46/Yr29 (Singh et al., 1998), Lr67/Yr46/Sr55 (Hiebert et al., 2010; Herrera-Fossel et al., 2011), Lr68 (Herrera-Foessel et al., 2012) and $\operatorname{Lr} 75$ (Singla et al., 2017). Some APR genes show race-specific responses, and these include $\operatorname{Lr} 12$ (McIntosh et al., 1995) and Lr22b (Dyck, 1979). While Lr48 and Lr49 were assigned by Saini et al. (2002) to the hypersensitive category based on monocyclic flag leaf tests, Bariana and Bansal (unpublished results) observed these genes to be slow rusting under polycyclic infection conditions in the field.

Traditionally, the development of new wheat cultivars has followed conventional phenotypic selection of desirable traits. Although this approach remains effective, it faces significant challenges due to the length of time taken to release a new cultivar (Forster et al., 2015). Recent advances in wheat genomics have led to the development of more efficient and precise approaches for wheat improvement (Dubcovsky and Dvorak, 2007; Bariana et al., 2013). For example, the identification of DNA markers linked with rust resistance genes has largely overcome the limitations of phenotypic selection for pyramiding two or more genes in breeding programs (Bariana et al., 2007; Bariana and Bansal, 2017). Similarly, the availability of high-density genotyping platforms such as DArTseq (Diversity Arrays Technology, Bruce, Australia; Cruz et al., 2013) and Infinium iSelect 90K SNP bead chip array (Wang et al., 2014) have expedited the mapping of economic traits (Bariana and Bansal, 2017). The development of simple gel-free marker genotyping systems such as kompetitive allele-specific PCR (KASP; LGC Genomics, UK) have encouraged marker assisted selection in breeding programs.
The rate for development of trait-linked DNA markers has also been accelerated by the increasing availability of genomic resources and tools supporting high throughput genomics. For example, methods have been developed to isolate specific chromosomes using flow cytometry (Vrána et al., 2000; Giorgi et al., 2013), which can then be sequenced to interrogate individual chromosome DNA code. Such approaches are particularly useful in polyploid species such as wheat, because they not only reduce the genome complexity to a single chromosome but also eliminate problems associated with presence of homoeologous genomes for sequence assembly (Doležel et al., 2007). The ability to sequence individual chromosomes adds a new dimension to marker development and gene cloning in allopolyploids including wheat (Doležel et al., 2012; International Wheat Genome Sequencing Consortium (IWGSC), 2014).

An Indian cultivar VL404 (Kentana/Bungulla//Frontana/ General-Urquiza/3/ST464/PI-74106) was released in 1973 by Vivekananda Parvatiya Krishi Anusandhan Sansthan, Almora. This cultivar was susceptible at the two-leaf stage but showed resistance at the flag-leaf stage against Indian Pt pathotypes in monocyclic inoculations and the underlying resistance locus was formally named Lr49 (Saini et al., 2002). Lr49 was mapped on the long arm of chromosome 4B (Bansal et al., 2008) using the VL404/WL711 RIL population, however, the flanking markers were not sufficiently close for efficient marker assisted selection. The aim of this study was to fine map the gene Lr49 using recently published assembled reference genome sequence for variety Chinese Spring (Appels et al., 2018) and flow sorted chromosome 4B sequence for VL404 and WL711. This is a first step towards cloning the causal gene for Lr49 and developing a diagnostic marker for use in marker assisted selection.

\section{MATERIALS AND METHODS}

\section{Plant and Pathogen Materials}

The VL404/WL711 $\mathrm{F}_{6: 8}$ derived recombinant inbred line (RIL) population used in this study comprised 181 lines. Pedigree information for both parents is described in the earlier study (Saini et al., 2002). A diverse set of Australian (75) and European (90) wheat cultivars was used to test the strength of linkage between markers developed in this study and Lr49. DNA was extracted from each wheat line using the method described in Bansal et al. (2014a).

\section{Greenhouse Tests}

Eight to 10 seeds of each RIL and parents were sown in 9-cm diameter pots as four lines per pot. Twenty grams of complete fertilizer Aquasol dissolved in 10L of tap water was applied to pots filled with potting mix before sowing. Plants were grown to the $4^{\text {th }}$ leaf stage at $20^{\circ} \mathrm{C}$ in a rust-free microclimate room prior to inoculation. Urea was applied every week prior to inoculation with Lr49-avirulent Pt pathotype 76-1,3,5,10,12 (culture no 539). The inoculation procedure described in Bansal et al. (2008) was followed. Rust response assessments were made 18 to 20 days post-inoculation using the infection type (IT) scale detailed in 
McIntosh et al. (1995). Briefly 0-4 infection type scale was used and RILs classified $<3$ were considered resistant and $>3$ susceptible.

\section{Sorting and Sequencing of Chromosome 4B}

Suspensions of intact mitotic chromosomes were prepared from synchronised root meristems of parental lines VL404 and WL711 (Vrána et al., 2000; Vrána et al., 2012) and GAA microsatellites on the chromosomes were labeled in suspension by fluorescein isothiocyanate (FITC) using the protocol for Fluorescence In Situ Hybridization In Suspension (FISHIS) (Giorgi et al., 2013). Genomic DNA was stained by 4',6diamidino2-phenylindole (DAPI) and chromosomes were analyzed on a FACSAria II SORP high speed flow sorter (BD Biosciences, San José, USA) as described in Kubaláková et al. (2002). One thousand chromosomes from each cluster were sorted onto a microscopic slide into a drop of $10 \mu \mathrm{l}$ of PRINS buffer supplemented with 5\% sucrose (Kubaláková et al., 1997). To assign chromosomes to an individual cluster on a dot plot, FISH with probes for GAA and Afa repeats was used to identify flow-sorted chromosomes and to assess purity. Chromosomal DNA of $4 \mathrm{~B}$ was amplified by the Multiple Displacement Amplified (MDA) approach using the Illustra GenomiPhi V2 DNA amplification kit (GE Healthcare, http://www.gehealthcare.com) as described in Šimková et al. (2008). Sequencing libraries were generated using the Nextera DNA sample preparation kit (Illumina Inc, San Diego, CA, USA) and 50 ng of DNA was amplified (according to the manufacturer's instructions, with the exception for usage of 3 $\mu \mathrm{l}$ of TDE1 for DNA fragmentation). Libraries with insert sizes of 600-800 bp were selected for sequencing. The insert sizes were verified using the Agilent DNA 1000 Kit (Agilent Technologies, Inc.) and concentrations were assessed by the KAPA Library Quantification Kit (Kapa Biosystems, Woburn, USA). The libraries were sequenced as paired-end reads using the HiSeq Rapid SBS Kit v2 (2x250 bp) (Illumina Inc, San Diego, CA, USA).

\section{SNP Detection Using Flow Sorted Chromosome 4B Sequence}

GYDLE software (Gydle Inc. Bioinformatics Service, Quebec City, Canada; http://www.gydle.com) was used to quality filter the raw sequence reads (minimum phred score 20; minimum read length $50 \mathrm{bp}$ ) derived from flow-sorted $4 \mathrm{~B}$ chromosome sequences of VL404 and WL711 and to align the filtered reads to the International Wheat Genome Sequencing Consortium (IWGSC) reference genome sequence assembly for cultivar Chinese Spring (RefSeq assembly v1.0; Appels et al., 2018). Gydle software performs an exhaustive alignment search to guarantee each paired-end read is aligned at its best mapping position, providing the alignment score that exceeds $80 \%$ sequence homology. Paired-end reads that align to multiple positions with equal scores are randomly distributed across those positions to ensure that all alignment positions are fully and correctly reflected. The SNP variant discovery and genotype calling was performed using the aligned paired-end sequence reads for WL711 and VL404 and GYDLE "findsnp" function.

\section{DNA Genotyping}

Microsatellite markers previously reported to be linked with $\operatorname{Lr} 49$ (Bansal et al., 2008) were genotyped on the entire RIL population and parents following the amplification conditions described in Bansal et al. (2014b). Infinium iSelect 90K SNP genotyping was performed on 12 resistant and 12 susceptible RILs, as reported in Wang et al. (2014). Genotype calling was performed using GenomeStudio (Illumina) and a custom perl script to assign genotype calls. Closely linked 90K SNPs and those identified from the flow-sorted chromosome sequences were converted into kompetitive allele-specific PCR (KASP) assays (LGC Genomics) following the manufacturers guidelines. For each KASP marker, two allele-specific forward primers and one common reverse primer were designed using BatchPrimer3 v1.0 (https://wheat.pw.usda.gov/demos/BatchPrimer3/) software. The PCR reaction contained $3 \mu \mathrm{l}$ of DNA $(30 \mathrm{ng} / \mu \mathrm{l})$, $5 \mu \mathrm{l}$ KASP mix (LGC Biosearch Technologies), and $0.11 \mu \mathrm{l}$ of primer mix $(12 \mu \mathrm{M}$ of each allele specific primer and $30 \mu \mathrm{M}$ of reverse primer). Reaction was performed in CFX96 real time PCR machine (Biorad, USA) with the following cycling conditions: $15 \mathrm{~min}$ at $94^{\circ} \mathrm{C} ; 10$ touchdown cycles of $20 \mathrm{~s}$ at $94^{\circ}$ $\mathrm{C}, 60 \mathrm{~s}$ at $65-57^{\circ} \mathrm{C}$ (dropping $0.8^{\circ} \mathrm{C}$ per cycle); and $26-35$ cycles of $20 \mathrm{~s}$ at $94^{\circ} \mathrm{C}, 60 \mathrm{~s}$ at $57^{\circ} \mathrm{C}$. Flourescence reading was taken at $40^{\circ} \mathrm{C}$ for $30 \mathrm{~s}$ and were analysed using allelic discrimination function. The KASP markers derived from 90K SNPs were named with the prefix KASP, followed by a number corresponding to the SNP index on the Infinium bead chip. The KASP markers derived from flow-sorted chromosome sequence variants were designated by the prefix sunKASP ( sun $=$ Sydney University) followed by a consecutive number.

\section{High Resolution Mapping}

VL404 was crossed with Avocet S to develop a high-resolution mapping population consisting of $2560 \mathrm{~F}_{2}$ plants. DNA was extracted from each $\mathrm{F}_{2}$ plant and tested with the Lr49 flanking markers. Plants showing recombination between the flanking markers were transplanted and the high resolution $\mathrm{F}_{3}$ family was generated and phenotyped with $\mathrm{Pt}$ pathotype 76-1,3,5,10,12 at the $4^{\text {th }}$ leaf stage.

\section{Data Analyses and Genetic Mapping}

The RIL population was categorized as homozygous resistant (HR) or homozygous susceptible (HS) based on the phenotypic scores of parents. Chi-squared $\left(\chi^{2}\right)$ test was used to determine the goodness of fit of the observed segregation to the expected genetic ratios. Alleles for SNP and SSR markers were scored as A and B for parents VL404 and WL711, respectively.

A genetic map was generated using MapManager Version QTXb20 (Manly et al., 2001) and the Kosambi map function (Kosambi, 1943). The linkage map was drawn according to Voorrips (2002). A likelihood of odds (LOD) score of 3.0 was used as the threshold for declaring linkage among loci. 
The genetic-physical map viewer Pretzel (Keeble-Gagnère et al., 2019) was used to identify and visualize structural variation in the genomic region containing the Lr49 locus.

\section{RESULTS}

\section{Genetic Analysis}

VL404 (Lr49) and WL711 exhibited infection type (IT) X (mesothetic response which includes more than two infection types on the same leaf) and IT3+, respectively, when inoculated with $\mathrm{Pt}$ pathotype $76-1,3,5,10,12$ at the $4^{\text {th }}$ leaf stage under greenhouse conditions. Leaf rust test on VL404/WL711 RIL population revealed clear segregation of $\operatorname{Lr} 49$ (99 resistant: 82 susceptible; $\chi_{1: 1}^{2}=1.59$, nonsignificant at $P=0.05$ and $1 d . f$.).

\section{Chromosome Sorting and Sequencing}

Thirty-five thousand copies of chromosome 4B from each of VL404 and WL711 were sorted with 97\% and 98\% purity, respectively, and amplified by MDA. To minimize the risk of representation bias, the products from three independent MDA reactions were pooled. The amplification and pooling of $4 \mathrm{~B}$ chromosomal DNA from VL404 and WL711 yielded 7.88 and $8.36 \mu$ g DNA, respectively, and paired-end sequencing provided $70,519,221$ and 70,685,036 reads. Following quality filtering, the filtered reads were aligned to the reference genome sequence assembly of cultivar Chinese Spring. This resulted in 68.8 and $64.7 \%$ of the filtered reads uniquely mapping to chromosome $4 \mathrm{~B}$, representing 16.5 and 17.3 fold coverage for VL404 and WL711, respectively.

\section{Molecular Mapping}

Forty-five SNPs from the iSelect 90K Infinium array showed linkage with Lr49 and were converted into single-locus KASP assays. Twenty-one KASP markers that clearly discriminated the parents (Table 1) were genotyped on the entire RIL population and integrated into the previously reported microsatellite marker-based genetic linkage map carrying Lr49 (Figure 1). The closest proximal marker KASP_54629 mapped $2.7 \mathrm{cM}$ from Lr49, whereas the closest distal markers at $0.6 \mathrm{cM}$ and included several co-segregating KASP markers.

To further increase marker density for the $L r 49$ region, SNP discovery was performed using the flow-sorted chromosome sequences for VL404 and WL711. Twenty-eight SNPs with approximately even physical spacing were selected to span the Lr49 region and converted into KASP assays. Seven of these markers (Table 1) produced clear parental clusters and could be unambiguously scored on the RIL population. Integration of these KASP markers into the genetic map for the Lr49 region, resulted in sunKASP_21 mapping at $0.4 \mathrm{cM}$ proximal and sunKASP_24 (clustered with five additional KASP markers derived from 90K SNPs) at $0.6 \mathrm{cM}$ distal to $\operatorname{Lr} 49$ (Figure 1B).

TABLE 1 | Primer sequences for kompetitive allele-specific PCR (KASP) markers designed from SNP sequences that showed association with Lr49 on chromosome $4 \mathrm{BL}$ and SNPs discovered from the sqeuences of flow sorted chromosome 4B of parental genotypes.

\begin{tabular}{|c|c|c|c|c|}
\hline KASP marker & SNP ID & Forward (Allele 1) primer & Forward (Allele 2) primer & Common/reverse primer \\
\hline KASP_5827 & IWB5827 & gaagcagctggcagcactca & gaagcagctggcagcactcg & gctcagcctcaaggtcggtgtt \\
\hline$K A S P \_7042$ & IWB7042 & tccaagttgactcaagagacgaga & ccaagttgactcaagagacgagg & cctcctacgcaacaaccgacacaa \\
\hline$K A S P \_7783$ & IWB7783 & agtaagaggcactaccgttcagatt & aagaggcactaccgttcagatc & agagggcgtgctttccaagtgaat \\
\hline KASP_8082 & IWB8082 & ttgctggtctttagaaatccctc & gctttgctggtctttagaaatcccta & cttgcactaacatcacaacccccat \\
\hline KASP_8302 & IWB8302 & acccttttacaacaacttcattcgc & ctacccttttacaacaacttcattcgt & gggaataatcagggattgaccccta \\
\hline$K A S P \_8708$ & MB8708 & ggtcgtggtgacgcacgcaa & gtcgtggtgacgcacgcag & ttcacatgaccatggccaggagat \\
\hline KASP_8980 & IWB8980 & agagcagaattgattgctgcaact & agagcagaattgattgctgcaacc & ctccacttcctcactgtcatctgtt \\
\hline$K A S P \_12434$ & IWB12434 & cgcacgacgagtgctgcca & gcacgacgagtgctgccc & gtcaaggtcgaccaaccctgaa \\
\hline KASP_20288 & IWB20288 & gtcctcaatttggagatgcgctt & gtcctcaatttggagatgcgctg & atcaagatgtaattctggattcagcaagaa \\
\hline KASP_20289 & IWB20289 & tcttttatactcacattctgaatcaggat & cttttatactcacattctgaatcaggac & ctccgagagtcaaaatacaagaagtgttt \\
\hline KASP_21440 & IWB21440 & gtccccagctaatcctgtggaa & ccccagctaatcctgtggag & tagtttctgtagcttcggttgatacctt \\
\hline KASP_26845 & IWB26845 & cagtttaatatgcagcagcactta & ctcagtttaatatgcagcagcacttc & gacgcagatccatctcagaaggtaa \\
\hline KASP_35049 & IWB35049 & aagcgaaagagaaactatttacagt & ctaagcgaaagagaaactatttacagc & tttagacacagcgatacgttgtacatgtt \\
\hline KASP_36379 & IWB36379 & gtgaccttatgactggtagaag & cctgtgaccttatgactggtagaaa & aaatcgcaatttsaacatgaattcagtctt \\
\hline KASP_39484 & IWB39484 & agtcaatgcaaggaggagaaa & ctagtcaatgcaaggaggagaag & gccgcttgttaggcttctggctt \\
\hline KASP_53740 & IWB53740 & tgtcatcttcattttcagcatctgca & gtcatcttcattttcagcatctgcg & gatccagaggagaaaggttctgcta \\
\hline KASP_54629 & IWB54629 & gtgtctccaagtgacagttgaatgt & gtctccaagtgacagttgaatgc & atccagcttcttgtacagcggagaa \\
\hline KASP_54728 & IWB54728 & gatgacatcgacggcgaactga & atgacatcgacggcgaactgg & acaccggctggtatgccaccaa \\
\hline KASP_59160 & IWB59160 & aagatttgctttcgatccgtacttca & agatttgctttcgatccgtacttcg & gtaacaacgattcaaatgtggacacgaaa \\
\hline KASP_72709 & IWB72709 & cgatcctaatgaaccgacgtattgt & gatcctaatgaaccgacgtattgc & accgtagctgacttggttgcagaa \\
\hline KASP_1109 & IWB1109 & tattcatcttacgattcttaaatacttccaat & catcttacgattcttaaatacttccaac & ccagggttgtgtgccttcctttatt \\
\hline sunKASP_21 & - & gattcgaatgtttttgtaggatttc & ttcagatctaaaatcacggcact & ctattaacgtagagcccagtgc \\
\hline sunKASP_24 & - & ttcgattacccgggagc & ttcgattacccgggagt & tgggttaagggcaagaaaca \\
\hline sunKASP_26 & - & agtaccaaatgcagcaaaaaaa & cagtaccaaatgcagcaaaaaat & ctttggcccaagttgtgtct \\
\hline sunKASP_31 & - & tcaatcatttactttcatgcgag & aatgtaattttattttgttttgcttgc & caccgaccaccattgttcta \\
\hline sunKASP_33 & - & catgtcaatagttatgcactcaaattg & aatctttttgctagccttcatctc & tggtccaagtacaggtctacca \\
\hline sunKASP_35 & - & caaatcctaaaagccaagatgc & ttcatttcgggactggga & cggagctatttttggaccag \\
\hline sunKASP_39 & - & caccatctcctcctcattatca & catcctctagaacaatggtggtc & ctcttcccgttgcaagaaat \\
\hline
\end{tabular}

Allele 1 primer labeled with FAM: GAAGGTGACCAAGTTCATGCT;

Allele 2 primer labeled with HEX: GAAGGTCGGAGTCAACGGATT. 


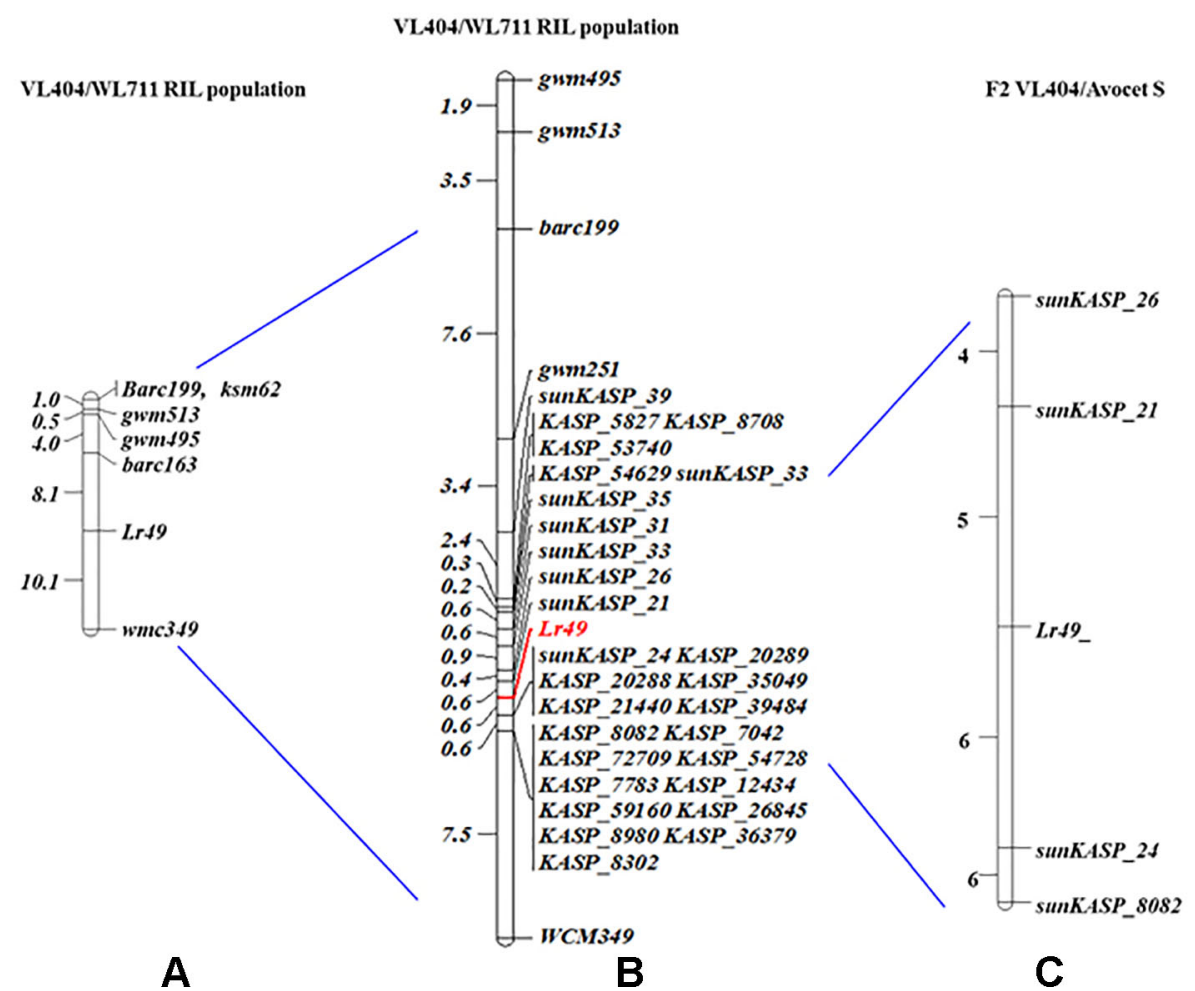

FIGURE 1 | Genetic linkage map of chromosome 4BL for the VL404/WL711 RIL population (A) Bansal et al. (2008), (B) present study (resistance gene is shown in red text), and (C) high resolution map of VL404/Avocet S (number of recombinants is given on the left).

\section{Construction of High-Resolution Map}

Markers sunKASP_21, sunKASP_26, sunKASP_24 and KASP_8082 that flanked Lr49 were genotyped on 2560 VL404/ Avocet $\mathrm{S}_{2}$ plants to identify recombinants for the construction of a high-resolution map. Twenty-one recombinants were observed. The recombinant $\mathrm{F}_{3}$ families were scored as homozygous resistant, homozygous susceptible and segregating. Five and six recombinants were observed between Lr49 and markers sunKASP_21 and sunKASP_24, respectively (Figure 1C).

\section{Assessing Marker Linkage Using Unrelated Materials}

Flanking markers sunKASP_21, sunKASP_24, KASP_20289, KASP_20288, KASP_35049, KASP_21440 and KASP_39484 were genotyped on a diverse set of 75 Australian and 90 European cultivars, unlikely to carry $L r 49$, to test the marker linkage with Lr49. Across the diverse germplasm, the proximal marker sunKASP_21 showed the strongest linkage, amplifying the susceptible WL711 (T:T) allele in all cultivars, except Gazelle, Safir and JO 8023, which amplified the resistance VL404 allele (C:C) (Table 2). In contrast, the distal markers (sunKASP_24, KASP_20289, KASP_20288, KASP_35049, KASP_21440 and $\left.K A S P \_39484\right)$ showed poor linkage, amplifying both the resistant and susceptible alleles (data not shown).

\section{Identification Closely Linked Markers}

Exome SNPs from 890 globally diverse accessions (He et al., 2019) located within the $L r 49$ region were used to identify recombination hot spots distal to marker sunKASP_21. The physical order of SNPs across the $L r 49$ region were used to identify five major haplotypes. VL404 belonged to one of these haplotypes and WL711 to another. Across the five haplotypes, two recombination sites were observed distal to the physical mapping position of sunKASP_21 and proximal to those of the co-segregating markers (sunKASP_24, KASP_20289, KASP_20288, KASP_35049, KASP_21440 and KASP_39484) in the RIL population (Figure 2). The recombination sites explain the breakdown in linkage (many false positives) observed in the diverse panel of Australian and European cultivars for markers distal to Lr49.

To develop markers between the two recombination sites and sunKASP_21, 35 exome SNPs proximal to the recombination sites and distal to sunKASP_21 were converted into KASP markers. However, only two of the KASP markers (sunKASP_261 and sunKASP_282) were polymorphic between VL404 and WL711 and produced a scorable pattern. When genotyped on the RIL population and integrated into the genetic map, neither marker was closely linked to Lr49. Further, based on the physical position of sunKASP_282 in the reference genome assembly of cultivar Chinese Spring, the marker fell within a putative deletion in the haplotype corresponding to VL404 (Figure 2). No additional SNP from the 
TABLE 2 | Validation of closely linked kompetitive allele-specific PCR (KASP) marker on Australian and European wheat cultivars.

\section{Cultivars/lines}

SUnKASP_21

VL404 (Lr49)

WL711

AGT Katana, Axe, Baxter, Beaufort, Bolac, Calingiri, Carnamah, Catalina, Chara, Cobra, Corack, Correll, Crusader, Dart, Derrimut, Diamondbird, EGA

\section{$\mathrm{C}: \mathrm{C}$}

$\mathrm{T}: \mathrm{T}$

$\mathrm{T}: \mathrm{T}$

Bonnie Rock, EGA Bounty, EGA Burke, EGA Gregory, EGA Wedgetail, EGA Wylie, Elmore CL PLus, Emu Rock, Envoy, Espada, Estoc, Forrest, Fortune, Gauntlet, GBA Sapphire, Giles, Gladius, Grenade CL Plus, Impala, Impose CL Plus, Janz, Justica CL Plus, King Rock, Kord CL Plus, Kunjin, Lang, Lincoln, Livingston, Mace, MacKellar, Magenta, Merinda, Merlin, Naparoo, Orion, Phantom, Preston, Scout, Sentinel, Shield, Spitfire, SQP Revenue, Strzelecki, Sunco, Sunguard, Suntop, Sunvale, Sunvex, Sunzell, Ventura, Waagan, Wallup, Wedin, Westonia, Wyalkatchem, Wylah, Yandanooka, Yitpi, Young

Gazelle, JO 8023, Safir

Apu, Aros, Atson, Avle, Bastian, Bjarne, Blanka, Børsum, Boru, Canon, Dala, Dalarna, Diamant, Diamant II, Drabant, Dragon, ELS 6404 - 102 - 3 , Ergo, Eroica, Extra Kolben, Fagott, Fram I, Fram II, Fylgia I, Fylgia II, Haarajärvi ME0102 Apu, Halland, Horsmanaho ME201 Timantti, J-03, Järvenkylä ME0302 Timantti, JO 3524, Jokikylä ME0505 Apu, Kadett, Kärn II, Kenya Farmer, Kimmo, Kiuru, Kota, Laitiala AP0103, Landvårkveite, Lantvete från Dalarna, Lantvete från Halland, Lavett, Manu, Monola ME1301, Møystad, MS273-150, Naxos, Nemares, Nora, Norrøna, østby, Polkka, Pompe, Pondus, Prins, Progress, Rang, Reno, Ring, Rival, Rollo, Rubin, Runar, Ruso, Saffran, Sappo, Sibirian, Skirne, Snøgg II, Snøgg I, Sober, Sopu, Sport, Svenno, Timantti, Timantti Paavo, Tjalve, Touko, Troll, Trym, Ulla, Vinjett, Vitus, Walter, William, WW 20299, Zebra,

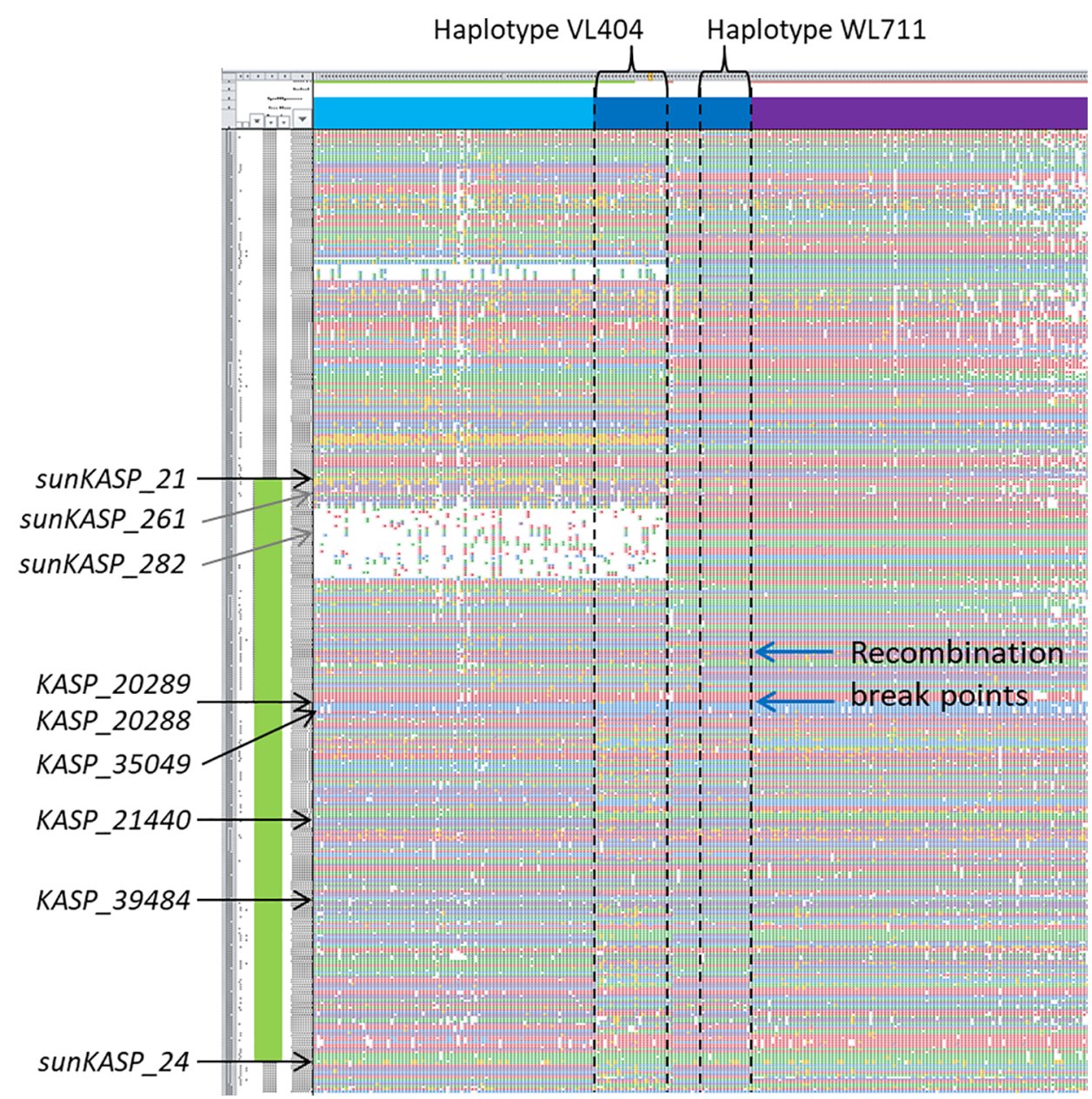

FIGURE 2 | Exome SNP genotypes for 890 globally diverse accessions across the Lr49 region (green box). The sunKASP_21 maps proximal to Lr49, while KASP_20289, KASP_20288, KASP_35049, KASP_21440, KASP_39484, and sunKASP_24 cosegregate and map distally to Lr49 in the RIL mapping population. Physical mapping positions for the flanking markers are indicated by black arrows. Two recombination breakpoints present between sunKASP_21 and KASP_20289 are shown by blue arrows. Two additional markers, sunKASP_261 and sunKASP_282, developed from parental SNPs proximal to the recombination break points and mapped in the RIL population were not linked to Lr49. The physical mapping position for these two markers is denoted by grey arrows. 
parental flow-sorted chromosome sequences could be identified for the region between sunKASP_21 and the two recombination sites.

\section{Genomic Structure of the Lr49 Region}

Comparison of marker loci order in the genetic linkage map for the VL404/WL711 RIL population with the physical positions of the same marker loci in the reference genome assembly for Chinese Spring revealed a complex pattern (Figure 3). It showed that the marker loci order distal to sunKASP_21 in the RIL population was colinear with Chinese Spring, while the marker loci order proximal to sunKASP_26 was inverted. The inversion was contained within the genomic region of Chinese Spring that appeared to be colinear with the RIL population distal to marker sunKASP_26. As repeated genotyping of the RIL population with the KASP markers produced the same result, the complex pattern suggested the presence of structural variation between one or both parents of the RIL population and Chinese Spring. Comparison of the physical map order of high confidence genes across the orthologous region in Ae. tauschii chromosome 4D, and homoeologous regions in Chinese Spring chromosome 4D
(IWGSCv1.0), emmer chromosome 4B (Avni et al., 2017) and Chinese Spring chromosome 4B (IWGSCv1.0) showed no evidence for structural rearrangements (Supplementary Figure 1), indicating that the structural variation was present in one or both parents and not Chinese Spring.

The genomic region between SunKASP_21 and the markers cosegregating with sunKASP_24 in the Chinese Spring reference genome assembly sequence contained 13 high confidence genes annotated in the IWGSC v1.0 genome release (Table 3). A gene containing a putative LRR motif (TraesCS4B01G301300) could be the likely candidate for Lr49. However, despite having good sequence read coverage in both VL404 and WL711, no nucleotide variation was identified in the coding sequence of the gene that would result in an asynonymous mutation or premature stop codon. This finding suggests that this gene is an unlikely candidate for $L r 49$, although differences in gene expression caused by a noncoding variant between the parent lines cannot be ruled out.

The alignment of the flow-sorted chromosome paired-end sequence reads from each parent to the reference genome

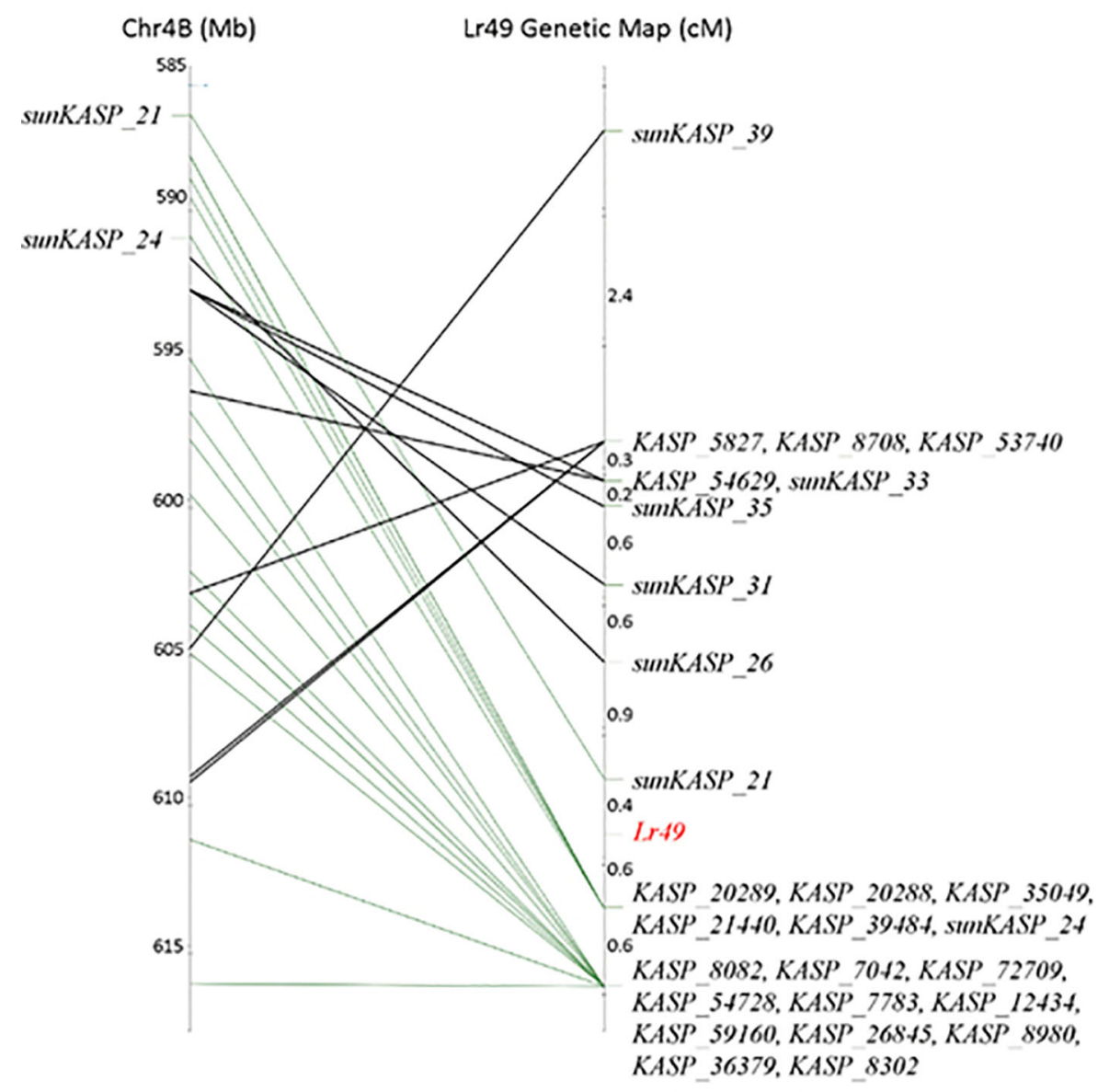

FIGURE 3 | Comparison of Lr49 genetic linkage map with the 585 to $617 \mathrm{Mbp}$ interval of Chinese Spring chromosome 4B physical map using Pretzel, a genetic map viewing software (http://plantinformatics.io). Markers distal to sunKASP_26 are colinear with the physical map (green connections). The genetic map order of markers proximal to sunKASP_21 does not reflect the physical map order in Chinese Spring (black connections). 
TABLE 3 | High confidence genes annotated in the IWGSC v1.0 genome sequence between flanking markers SunKASP_21 and markers cosegregating with sunKASP_24.

\section{IWGSC v1.0 Gene ID}

IWGSC v1.0 human readable gene annotation

TraesCS4B01G300700

TraesCS4B01G300800

TraesCS4B01G300900

TraesCS4B01G301000

TraesCS4B01G301100

TraesCS4B01G301200

TraesCS4B01G301300

TraesCS4B01G301400

TraesCS4B01G301500

TraesCS4B01G301600

TraesCS4B01G301700

TraesCS4B01G301800

TraesCS4B01G301900

TraesCS4B01G302000

TraesCS4B01G302100

TraesCS4B01G302200

TraesCS4B01G302300

TraesCS4B01G302400

TraesCS4B01G302500

TraesCS4B01G302600

TraesCS4B01G302700

TraesCS4B01G302800

TraesCS4B01G302900

TraesCS4B01G303000

TraesCS4B01G303100

TraesCS4B01G303200

TraesCS4B01G303300
Carotenoid cleavage dioxygenase

Tryptophan synthase beta chain

Peptide chain release factor 1

DNA-directed RNA polymerase subunit beta

Receptor-like protein kinase

Hexosyltransferase

Leucine-rich repeat receptor-like protein kinase

B3 domain-containing protein

$\mathrm{NAD}(\mathrm{P})$-binding Rossmann-fold superfamily protein

Origin recognition complex subunit 2

Transmembrane protein, putative

Alpha/beta-Hydrolases superfamily protein

Thionin-like protein

Agmatine coumaroyltransferase-2

Agmatine coumaroyltransferase-2

Agmatine coumaroyltransferase-2

Agmatine coumaroyltransferase-2

Uroporphyrinogen III synthase

Transmembrane protein 131

MADS box transcription factor

Vacuolar-sorting-associated protein 37-like protein

Aspartic proteinase nepenthesin-1

DNA-directed RNA polymerase subunit

Dentin sialophosphoprotein-related, putative isoform 1

WD and tetratricopeptide repeat protein, putative

Zinc finger protein
Ubiquitin carboxyl-terminal hydrolase 2 assembly of Chinese Spring using GYDLE software revealed the presence of two distinct sequence haplotypes in the susceptible parent WL711 that spanned the entire Lr49 region delineated by markers sunKASP_21 and sunKASP_36379. Indeed, these sequence haplotypes extended well beyond the Lr49 region both distally and proximally (Figure 4). In contrast, a single but different sequence haplotype was observed in the resistant parent VL404 within the Lr49 region delineated by markers sunKASP_21 and sunKASP_36379. Outside this region, VL404 possessed two distinct sequence haplotypes, one of which was identical to one of those observed in WL711 and the single sequence haplotype present in Chinese Spring, which was observed when Chinese Spring flow-sorted chromosome paired-end sequence reads were aligned to pseudomolecule $4 \mathrm{~B}$ (IWGSCv1.0) (Figure 4). The presence of only a single sequence haplotype within the Lr49 region delineated by markers sunKASP_21 and sunKASP_36379 in VL404, relative to WL711, was further supported by paired-end sequence read coverage across the interval (Supplementary Figure 2). These observations indicate the presence of structural chromosomal differences between VL404, WL711 and Chinese Spring.

\section{DISCUSSION}

Cultivars carrying long-lasting resistance to diseases have been released through conventional phenotypic screening; however, the genetic basis of resistance has been largely unknown. It is

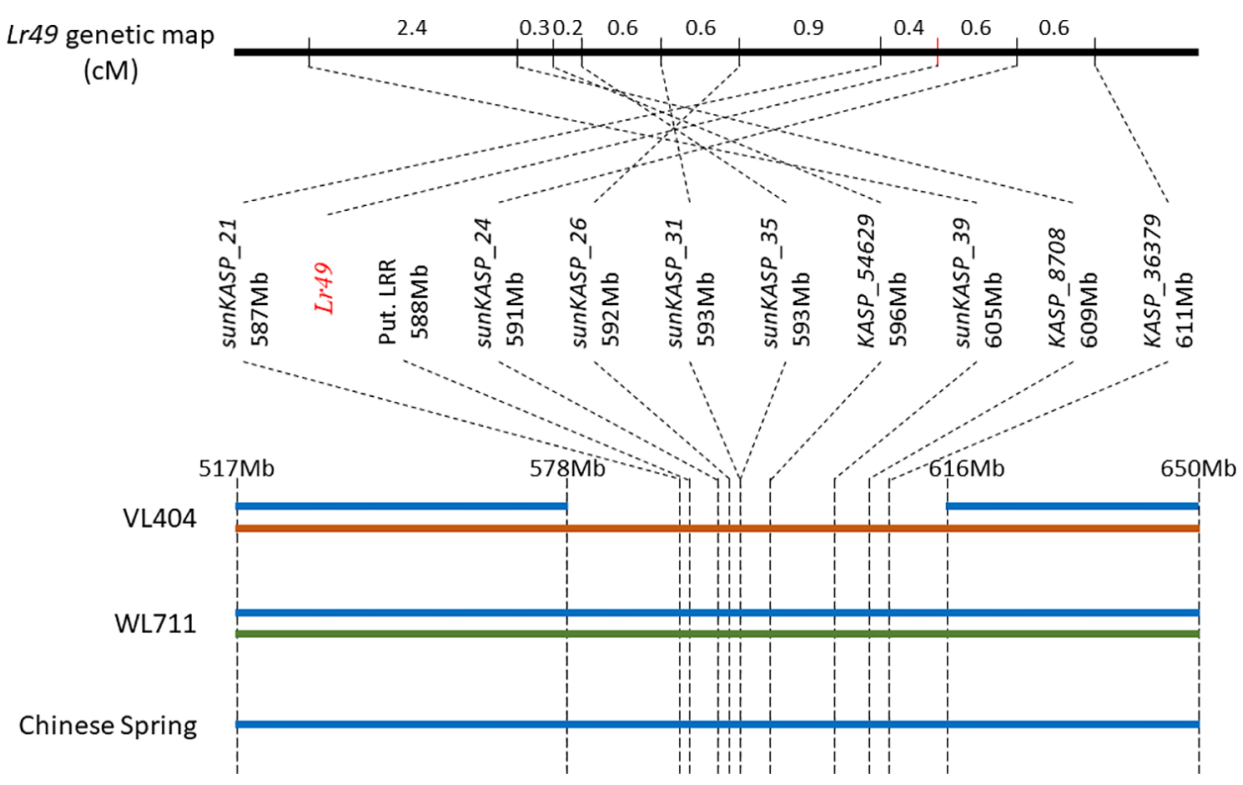

FIGURE 4 | Diagrammatic representation of the Lr49 interval and flanking region on chromosome $4 B$. Both VL404 (resistant parent) and WL711 (susceptible parent) have two sequence haplotypes outside the Lr49 region, while VL404 has only one sequence haplotype within the Lr49 interval. Chinese Spring has only one sequence haplotype across entire region. Each unique sequence haplotype is represented by a different color. Markers mapping within the $L r 49$ interval are ordered based on their physical mapping location. The Lr49 genetic map does not reflect the physical mapping order as illustrated by comparison of genetic and physical map order across the Lr49 interval. Differences in sequence haplotype structure and physical-genetic map order in VL404 and WL711, relative to Chinese Spring, suggests structural rearrangement or copy number variation (CNV) relative to Chinese Spring. 
challenging to combine ASR and APR genes with confidence into a single genotype using phenotypic assays, as the high level of resistance conditioned by effective ASR genes masks the detection of APR loci. Molecular markers linked with rust resistance genes developed through fine mapping can be used to pyramid resistance genes into a single genotype efficiently and reliably.

We used high-density genotyping and flow-sorted chromosome sequencing to fine-map the genomic region on chromosome 4B containing $L r 49$ as a first step towards developing a diagnostic marker for use in marker assisted selection and for cloning of this gene. Genetic mapping of VL404/WL711 RIL population using the iSelect 90K wheat SNP bead chip array localized Lr49 to a 3.3-cM interval. Subsequent mapping using KASP markers targeting nucleotide variation identified from flow-sorted chromosome sequences of the parental lines VL404 and WL711 that was expected to tile across the physical region corresponding to Lr49 in the Chinese Spring chromosome $4 \mathrm{~B}$ pseudomolecule, further reduced the Lr49 interval to $1.0 \mathrm{cM}$ (Figure 1).

While the availability of flow-sorted chromosome sequences for the parental lines VL404 and WL711 allowed the rapid identification of nucleotide variation to further fine map the Lr49 interval and to largely preclude a high-confidence gene containing a LRR motif as a candidate for Lr49, many of the KASP markers targeting this polymorphism could not be reliably scored in the mapping population. Indeed only seven of 28 KASP assays developed for this purpose could be genetically mapped. Comparison of the Lr49 genetic linkage map with the Chinese Spring chromosome 4B physical map (Figure 3), followed by visualization of the alignment of the flow-sorted chromosome paired-end sequence reads from each parent to the reference genome assembly of Chinese Spring (Figure 4), suggested structural variations in VL404 and WL711 compared to Chinese Spring. This was supported by observed collinearity of high confidence genes across the orthologous region in Ae. tauschii chromosome 4D, and homoeologous regions in Chinese Spring chromosome 4D, emmer chromosome 4B, and Chinese Spring chromosome 4B (Supplementary Figure 1). These observations suggested that the linkage map appeared inverted and overlapping when compared to the physical position of the markers in the Chinese Spring $4 \mathrm{~B}$ pseudomolecule (Figure 3), and the presence of two sequence haplotypes in WL711 across the Lr49 region and beyond the Lr49 interval in VL404 (Figure 4). These results implied the presence of an inverted local duplication in each of the parental lines relative to Chinese Spring. The presence of two distinct sequence haplotypes across the $L r 49$ region in the susceptible parent W711 and only one in the resistant parent VL404 suggested further localized structural variation between the parental lines (Figure 4). This structural variation is likely to explain the difficulty encountered for developing scorable KASP markers for polymorphism identified from the parental flow sorted chromosome sequence. KASP markers that assay multiple loci exhibit cluster compression due to the increase in allele dosage, and therefore are more difficult to score compared to a KASP marker that assays only a single locus. Cluster compression is expected for KASP markers that assay a duplicated genomic region. The presence of extensive local structural variation between wheat cultivars has been previously reported (Montenegro et al., 2017; Clavijo et al., 2017).

It is unclear whether the presence of only one haplotype for the Lr49 region in VL404 represents a structural deletion, or the presence of a chromosomal segment that is sufficiently diverged at the nucleotide level such that paired-end reads from this region in VL404 could not be aligned to the assembled genome sequence of Chinese Spring. As Chinese Spring does not carry Lr49, it is likely that VL404 carries a resistance gene absent in Chinese Spring. This scenario is possible since VL404 was derived through crosses involving durum wheat. We are currently using various sequencing technologies including Nextera mate-pair sequencing and Dovetail Genomics scaffolding technology (Thind et al., 2017) to de novo assemble the Lr49 region for VL404 and WL711, which will elucidate the physical structure and help to clone Lr49 and develop a diagnostic marker for use in breeding.

Marker sunKASP_21 showed the strongest association with Lr49 when tested on a diverse set of 75 Australian and 90 European cultivars, which were unlikely to carry Lr49. The marker amplified the VL404 allele in only three genotypes (Gazelle, JO 8023 and Safir) and the non-Lr49 associated alleles in the remaining 162 genotypes. This result was supported by the major SNP haplotypes for the Lr49 region revealed by the 890 globally diverse exome sequenced accessions, which showed evidence for two historical recombination sites distal to sunKASP_21 (Figure 2). The combination of these two pieces of evidence suggests that sunKASP_21 can be used for marker assisted deployment of Lr49 in breeding programs.

In conclusion, our study has demonstrated application of the assembled reference genome sequence for cultivar Chinese Spring and the flow-sorted chromosome sequences of the parental lines to accelerate fine-mapping of trait loci in common wheat. Our results also highlight the challenge for cloning Lr49 caused by the presence of structural variation in the parental lines of the mapping population, relative to Chinese Spring, and the importance of being able to assemble such regions to enable the cloning of causal genes and development of diagnostic markers for use in breeding.

\section{DATA AVAILABILITY STATEMENT}

The SNP data used in the study were previously generated and can be found in the European Nucleotide Archive using accession number ERZ805275 (https://www.ebi.ac.uk/ena/data/ view/ERZ805275).

\section{AUTHOR CONTRIBUTIONS}

VN conducted initial mapping of KASP markers and drafted the manuscript. DB developed high resolution population and tested 
flanking markers on it. MV and JD sorted chromosome 4B from parental lines and conducted sequencing. NQ mapped markers developed from the flow sorted chromosomes. $\mathrm{PK}, \mathrm{KF}$, and $\mathrm{MH}$ aligned flow sorted chromosome sequences with the reference sequence. UB developed KASP and sunKASP markers. PK, MH, $\mathrm{UB}$, and $\mathrm{HB}$ edited the manuscript. $\mathrm{UB}$ and $\mathrm{HB}$ provided overall supervision. All authors read the manuscript.

\section{FUNDING}

VN was funded by Australia Awards and the Ugandan Government. UB, HB, PK, KF and $\mathrm{MH}$ were funded through research grants from the Grains Research \& Development Corporation (GRDC) Australia. MV and JD were supported by

\section{REFERENCES}

Appels, R., Eversole, K., Feuillet, C., Keller, B., Rogers, J., Stein, N., et al. (2018). Shifting the limits in wheat research and breeding using a fully annotated reference genome. Science 361, 661. doi: 10.1126/science.aar7191

Avni, R., Nave, M., Barad, O., Baruch, K., Twardziok, S. O., Gundlach, H., et al. (2017). Wild emmer genome architecture and diversity elucidate wheat evolution and domestication. Science 357, 93-97. doi: 10.1126/science.aan0032

Bansal, U. K., Hayden, M. J., Venkata, B. P., Khanna, R., Saini, R. G., and Bariana, H. S. (2008). Genetic mapping of adult plant leaf rust resistance genes $L r 48$ and Lr49 in common wheat. Theor. Appl. Genet. 117, 307-312. doi: 10.1007/ s00122-008-0775-6

Bansal, U. K., Kazi, A. G., Singh, B., Hare, R. A., and Bariana, H. S. (2014a). Mapping of durable stripe rust resistance in a durum wheat cultivar Wollaroi. Mol. Breed 33, 51- 59. doi: 10.1007/s11032-013-9933-x

Bansal, U. K., Wicker, T., Keller, B., Hayden, M., and Bariana, H. S. (2014b). Molecular mapping of an adult plant stem rust resistance gene Sr56 in winter wheat cultivar Arina. Theor. Appl. Genet. 127, 1441-1448. doi: 10.1007/s00122014-2311-1

Bariana, H. S., and Bansal, U. K. (2017). "Breeding for disease Resistance," in Encyclopedia of applied plant sciences, vol. 3 . Eds. B. Kole, G. B. Murray and J. D. Murphy (Waltham, MA: Academic Press), 69-76.

Bariana, H. S., Brown, G. N., Bansal, U. K., Miah, H., Standen, G. E., and Lu, M. (2007). Breeding for triple rust resistance wheat cultivars for Australia using conventional and marker assisted selection technologies. Aust. J. Agric. Res. 58, 576-587. doi: 10.1071/AR07124

Bariana, H., Bansal, U., Basandrai, D., and Chhetri, M. (2013). "Disease Resistance," in Genomics and breeding for climate-resilient crops Vol. 2 Target Traits. Ed. C. Kole (Berlin, Germany: Springer-Verlag), 291-314.

Bariana, H. S. (2003). "Breeding for disease resistance," in Encyclopedia of applied plant sciences. Eds. B. Thomas, D. J. Murphy and B. G. Murray (UK: AcademicPress, Harcourt), 244-253.

Borlaug, N. E. (2007). Sixty-two years of fighting hunger: personal recollections. Euphytica 157, 287-297. doi: 10.1007/s10681-007-9480-9

Clavijo, B. J., Venturini, L., Schudoma, C., Accinelli, G. G., Kaithakottil, G., Wright, J., et al. (2017). An improved assembly and annotation of the allohexaploid wheat genome identifies complete families of agronomic genes and provides genomic evidence for chromosomal translocations. Genome Res. 27, 885-896. doi: 10.1101/gr.217117.116

Cruz, V. M., Kilian, A., and Dierig, D. A. (2013). Development of DArT marker platforms and genetic diversity assessment of the US collection of the new oilseed crop lesquerella and related species. PLoS ONE 8, e64062. doi: 10.1371/ journal.pone.0064062

Doležel J, Kubaláková M, Paux, E., Bartoš, J., and Feuillet, C. (2007). Chromosome-based genomics in the cereals. Chromosome Res. 15, 51-66. doi: $10.1007 / \mathrm{s} 10577-006-1106-\mathrm{x}$ the ERDF project "Plants as a tool for sustainable global development” (No. CZ.02.1.01/0.0/0.0/16_019/0000827).

\section{ACKNOWLEDGMENTS}

The authors thank Dr. Jan Vrána for chromosome sorting and Zdeňka Dubská and Romana Šperková for technical assistance.

\section{SUPPLEMENTARY MATERIAL}

The Supplementary Material for this article can be found online at: https://www.frontiersin.org/articles/10.3389/fpls.2019.01787/ full\#supplementary-material

Doležel J, Vrána J, Šafár, J., Bartoš, J., Kubaláková, M., and Šimková, H. (2012) Chromosomes in the flow to simplify genome analysis. Funct. Integr. Genomics 12, 397-416. doi: 10.1007/s10142-012-0293-0

Dubcovsky, J., and Dvorak, J. (2007). Genome plasticity a key factor in the success of polyploid wheat under domestication. Science 316, 1862-1866. doi: 10.1126/ science.1143986

Dyck, P. L. (1979). Identification of the gene for adult-plant leaf rust resistance in Thatcher. Can J. Plant Sci. 59, 499-501.

Forster, B. P., Till, B. J., Ghanim, A. M. A., Huynh, H. O. A., Burstmayr, H., and Caligari, P. D. S. (2015). Accelerated plant breeding. CAB Rev. 43, 1749-8848. doi: 10.1079/PAVSNNR20149043

Giorgi, D., Farina, A., Grosso, V., Gennaro, A., Ceoloni, C., and Lucretti, S. (2013). FISHIS: fluorescence in situhybridization in suspension and chromosome flow sorting made easy. PLoS One 8, e57994. doi: 10.1371/journal.pone.0057994

He, F., Pasam, R., Shi, F., Kant, S., Keeble-Gagnere, G., Kay, P., et al. (2019). Exome sequencing highlights the role of wild-relative introgression in shaping the adaptive landscape of the wheat genome. Nat. Genet. 51, 896-904. doi: 10.1038/s41588-019-0382-2

Herrera-Foessel, S. A., Singh, R. P., Huerta-Espino, J., Rosewarne, G. M., Periyannan, S. K., Viccars, L., et al. (2012). Lr68: a new gene conferring slow rusting resistance to leaf rust in wheat. Theor. Appl. Genet. 124, 1475-1486. doi: 10.1007/s00122-012-1802-1

Herrera-Fossel, S. A., Lagudah, E. S., Huerta-Espino, J., Hayden, M. J., Bariana, H. S., Singh, D., et al. (2011). New slow-rusting leaf rust and stripe rust resistance genes Lr67 and Yr46 in wheat are pleiotropic or closely linked. Theor. Appl. Genet. 122, 239-249. doi: 10.1007/s00122-010-1439-x

Hiebert, C. W., Thomas, J. B., McCallum, B. D., Humphreys, D. G., DePauw, R. M., Hayden, M. J., et al. (2010). An introgression on wheat chromosome 4DL in RL6077 (Thatcher ${ }^{*} 6$ / PI 250413) confers adult plant resistance to stripe rust and leaf rust (Lr67). Theor. Appl. Genet. 121, 1083-1091. doi: 10.1007/s00122-010-1373-y

International Wheat Genome Sequencing Consortium (IWGSC). (2014). A chromosome-based draft sequence of the hexaploid bread wheat (Triticum aestivum). Science 345, 6194. doi: 10.1126/science. 1251788

Keeble-Gagnère, G., Isdale, D., Suchecki, R., Kruger, A., Lomas, K., Carroll, D., et al. (2019). Integrating past, present and future wheat research with Pretzel. bioRxiv. 4. doi: 10.1101/517953

Kolmer, J. A. (2005). Tracking wheat rust on a continental scale. Curr. Opin. Plant Biol. 8, 441-449. doi: 10.1016/j.pbi.2005.05.001

Kosambi, D. D. (1943). The estimation of map distances from recombination values. Ann Eugen. 12, 172-175. doi: 10.1111/j.1469-1809.1943.tb02321.x

Kubaláková, M., Macas, J., and Doležel, J. (1997). Mapping of repeated DNA sequences in plant chromosomes by PRINS and C-PRINS. Theor. Appl. Genet. 94, 758-763. doi: 10.1007/s001220050475

Kubaláková, M., Vrána, J., Č́íhalíková, J., Šimková, H., and Doležel, J. (2002). Flow Karyotyping and chromosome sorting in bread wheat (Triticum aestivum L.). Theor. Appl. Genet. 104, 1362-1372. doi: 10.1007/s00122-002-0888-2 
Manly, K. F., Cudmore, RHJr, and Meer, J. M. (2001). Map Manager QTX, crossplatform software for genetic mapping. Mamm. Genome 12, 930-932. doi: 10.1007/s00335-001-1016-3

McIntosh, R. A., Park, R. F., and Wellings, C. R. (1995). Wheat Rusts: An Atlas of Resistance Genes (East Melbourne, Australia: CSIRO Publications).

McIntosh, R. A., Dubcovsky, J., Rogers, J. W., Morris, C., Appels, R., and Xia, C. X. (2013). Catalogue of gene symbols for wheat: 2013-2014 supplement. KOMUGI Integr. Wheat Sci. Database. Available online at http://www.shigen.nig.ac.jp/wheat/ komugi/genes/symbolClassList.jsp (Accessed on 15 August 2015).

Montenegro, J. D., Golicz, A. A., Bayer, P. E., Hurgobin, B., Lee, H., Chan, C. K., et al. (2017). The pangenome of hexaploid bread wheat. Plant J. 90, 1007-1013. doi: $10.1111 /$ tpj.13515

Saini, R. G., Kaur, M., Singh, B., Sharma, S., Nanda, G. S., Nayar, S. K., et al. (2002). Lr48 and Lr49, novel hypersensitive adult plant leaf rust resistance genes in wheat (Triticum aestivum L.). Euphytica 124, 365-370. doi: 10.1023/A:1015762812907

Singh, R. P., Mujeebkazi, A., and Huerta-Espino, J. (1998). Lr46: a gene conferring slow rusting resistance to leaf rust in wheat. Phytopathology 88, 890-894. doi: 10.1094/PHYTO.1998.88.9.890

Singh, R. P. (1992). Association between gene Lr34 for leaf rust resistance and leaf tip necrosis in wheat. Crop Sci. 32, 874-878. doi: 10.2135/cropsci1992.0011183 X003200040008x

Singla, J., Lüthi, L., Wicker, T., Bansal, U., Krattinger, S. G., and Keller, B. (2017). Characterization of Lr75: a partial, broad-spectrum leaf rust resistance gene in wheat. Theor. Appl. Genet. 130, 1-12. doi: 10.1007/s00122-016-2784-1

Šimková, H., Svensson, J. T., Condamine, P., Hřibová, E., Suchánková, P., Bhat, P. R., et al. (2008). Coupling amplified DNA from flow-sorted chromosomes to high-density SNP mapping in barley. BMC Genomics 9, 294. doi: 10.1186/ 1471-2164-9-294
Thind, A. K., Wicker, T., Šimková, H., Fossati, D., Moullet, O., Brabant, C., et al. (2017). Rapid cloning of genes in hexaploid wheat using cultivar-specific longrange chromosome assembly. Nat. Biotechnol. 35, 793-796. doi: 10.1038/ nbt.3877

Voorrips, R. E. (2002). MapChart: software for the graphical presentation of linkage maps and QTLs. J. Hered 93, 77-78. doi: 10.1093/jhered/93.1.77

Vrána, J., Kubaláková, M., Šimková, H., Č́íhalíková, J., Lysák, M. A., and Doležel, J. (2000). Flow-sorting of mitotic chromosomes in common wheat (Triticum aestivum L.). Genetics 156, 2033-2041.

Vrána, J., Šimková, H., Kubaláková, M., Č́ihalíková, J., and Doležel, J. (2012). Flow cytometric chromosome sorting in plants: the next generation. Methods 57, 331-337. doi: 10.1016/j.ymeth.2012.03.006

Wang, S., Wong, D., Forrest, K., Allen, A., Chao, S., Huang, B. E., et al. (2014). Characterization of polyploid wheat genomic diversity using a high-density 90,000 single nucleotide polymorphism array. Plant Biotechnol. J. 12, 787-796. doi: $10.1111 /$ pbi. 12183

Conflict of Interest: The authors declare that the research was conducted in the absence of any commercial or financial relationships that could be construed as a potential conflict of interest.

Copyright (C) 2020 Nsabiyera, Baranwal, Qureshi, Kay, Forrest, Valárik, Doležel, Hayden, Bariana and Bansal. This is an open-access article distributed under the terms of the Creative Commons Attribution License (CC BY). The use, distribution or reproduction in other forums is permitted, provided the original author(s) and the copyright owner(s) are credited and that the original publication in this journal is cited, in accordance with accepted academic practice. No use, distribution or reproduction is permitted which does not comply with these terms. 